\title{
Elevation of methylated DNA in KILLIN/PTEN in the plasma of patients with thyroid and/or breast cancer
}

This article was published in the following Dove Press journal:

OncoTargets and Therapy

II November 2014

Number of times this article has been viewed

\author{
Enders $\mathrm{K} \mathrm{Ng}{ }^{\prime}$ \\ Vivian Y Shin' \\ Candy $\mathrm{P}$ Leung' \\ Vivian W Chan ${ }^{2}$ \\ Fian B Law ${ }^{2,3}$ \\ Man T Siu' \\ Brian H Lang' \\ Edmond $\mathrm{S} \mathrm{Ma}^{2,3}$ \\ Ava Kwong ${ }^{1,3}$ \\ 'Department of Surgery, The \\ University of Hong Kong, \\ ${ }^{2}$ Department of Molecular Pathology \\ and Department of Surgery, Hong \\ Kong Sanatorium and Hospital, \\ ${ }^{3}$ Hong Kong Hereditary Breast \\ Cancer Family Registry, Hong Kong
}

Correspondence: Ava Kwong

Division of Breast Surgery, Queen Mary and Tung Wah Hospital, The University of Hong Kong, Pokfulam, Hong Kong Tel +85222554773

Fax +85228172291

Email akwong@asiabreastregistry.com

\begin{abstract}
Around $80 \%$ of mutations in the PTEN gene have been reported to be associated with diseases such as Cowden syndrome, which is an autosomal dominant disorder associated with an increased risk of developing breast, thyroid, and endometrial neoplasms. Recent studies have also demonstrated that KILLIN, which is located proximally to PTEN, shares the same transcription start site, and is assumed to be regulated by the same promoter, but is transcribed in the opposite direction. In this regard, we postulate that there may be a connection between KILLIN/PTEN genes and breast and thyroid cancers. Using real-time quantitative polymerase chain reaction (qPCR), we found that expression of KILLIN, but not PTEN, was significantly decreased in 23 Chinese women with a personal history of breast and thyroid cancer or a personal history of breast cancer and a family history of thyroid cancer, or vice versa, and at least two persons in the family with thyroid cancer or at a young age $<40$ years, when compared with healthy controls $(P<0.0001)$. No PTEN mutations were found in these 23 patients. We then developed a simple methylation-sensitive restriction enzyme digestion followed by real-time quantitative assay to quantify plasma methylated KILLIN/PTEN DNA in these patients. Plasma levels of methylated KILLIN/PTEN DNA were significantly increased in these patients when compared with healthy controls $(P<0.05)$. This study shows that plasma methylated KILLIN/ PTEN DNA was significantly elevated, suggesting hypermethylation of the KILLIN/PTEN promoter in breast and thyroid cancer patients.
\end{abstract}

Keywords: KILLIN, PTEN, hypermethylation, breast cancer, thyroid cancer

\section{Introduction}

Germline mutations in PTEN (phosphate and tensin homologue) have been reported to be associated with diseases such as Cowden syndrome (CS), and account for $80 \%$ of cases. ${ }^{1} \mathrm{CS}$ is an autosomal dominant disorder characterized by multiple hamartoma syndromes, and is associated with an increased risk of developing breast, thyroid, and endometrial neoplasms. ${ }^{1}$ Individuals who met at least the relaxed International Cowden Consortium operational criteria were recruited. Relaxed criteria are defined as full criteria minus one criterion, and such individuals are referred to as CS-like. The lifetime risk of breast cancer in CS patients is estimated to be in the range of $25 \%-50 \%$, with a pathological predominance of ductal and lobular carcinoma. ${ }^{1,2}$ Thyroid cancer is another common malignancy in patients with CS, with a lifetime risk of $10 \%$, and the follicular-derived type is most often observed. ${ }^{1-3}$

The PTEN gene spans $105 \mathrm{~kb}$ and contains nine exons on chromosome 10q23.31. It is a well characterized tumor suppressor gene that antagonizes the phosphoinositol3-kinase/protein kinase B (Akt) signaling pathway. The decreased level of phosphorylated Akt results in G1 cell cycle arrest and apoptosis. ${ }^{3,4}$ PTEN also regulates 
interactions between the cell and extracellular matrix via interaction with focal adhesion kinase. ${ }^{5}$ In addition to $\mathrm{CS}$, PTEN mutation is also reported in other hamartoma tumor syndromes, including Bannayan-Riley-Ruvalcaba syndrome, Proteus syndrome, and Proteus-like syndrome, as well as macrocephaly and autism. ${ }^{1,4,6}$

While the genetic predisposition of PTEN to multiple hamartoma syndromes in Caucasian populations is being increasingly understood, there are few relevant reports in Asian cohorts. ${ }^{7,8}$ Recent studies have reported a newly identified gene, KILLIN (RefSeq, NM_001126049), which is also located in the 10q23.31 chromosomal region, proximal to PTEN. Similar to PTEN, KILLIN is involved in cell cycle arrest and is regulated by p53. ${ }^{9,10}$ Interestingly, PTEN and KILLIN share the same transcription start site, and are assumed to be regulated by the same promoter, but are transcribed in opposite directions. Bennett et al recently demonstrated that approximately $30 \%$ of CS and CS-like patients without PTEN mutations had germline hypermethylation and downregulation of the KILLIN gene. ${ }^{10}$ Therefore, in this study, we sought to determine if there is any association between KILLIN/PTEN genes in patients with breast and/or thyroid cancer. We also investigated whether KILLIN/PTEN promoter hypermethylation and downregulation were present in the plasma of Chinese patients.

\section{Materials and methods \\ Patients}

Twenty-three Chinese women with breast and/or thyroid cancer and a family history of thyroid cancer were recruited from the Hong Kong Hereditary Breast Cancer Family Registry between March 1, 2009 and February 28, 2011. We included four patients with breast cancer only, three patients with thyroid cancer only, and 16 patients with breast and thyroid cancer. In our study cohort, none of the patients with both breast and thyroid cancer had a family history of thyroid cancer. Twenty healthy control subjects with no diagnosed malignancy were also recruited for the study. Blood samples were collected from patients at diagnosis or during surgery. All patients were selected for Chinese ancestry and met the criteria for genetic/familial high-risk assessment according to the National Comprehensive Cancer Network. All the patients with breast cancer were confirmed to be $B R C A 1 / 2$ mutation-negative by direct bidirectional sequencing and by multiplex ligation-dependent probe amplification testing. ${ }^{11,12}$ Written informed consent was obtained from all the participants, and the study was approved by the institutional review board of the University of Hong Kong/Hospital
Authority West Cluster and other contributing hospitals in Hong Kong.

\section{PTEN mutation screening by conventional DNA sequencing}

Mutation screening was done by direct bidirectional DNA sequencing of all coding exons for PTEN and partial flanking intronic sequences. All primer sequences are listed in Table S1. Mutation detection was performed on genomic DNA extracted from peripheral blood samples using a Qiagen DNA Mini blood kit (Qiagen, Hilden, Germany) according to the manufacturer's instructions. Bidirectional sequencing was performed using a BigDye ${ }^{\circledR}$ Terminator v3.1 cycle sequencing kit (Applied Biosystems, Foster City, CA, USA) and analyzed on an ABI 3130xl genetic analyzer (Applied Biosystems). The results of sequencing were compared with the reference DNA sequences using Variant Reporter software (Applied Biosystems) and then reviewed manually. Computational analysis for potential cryptic splice site mutation was performed using splice site prediction programs (NNSPLICE and ESEF finder) when sequence changes were identified. All mutation and sequence variants were named according to the description of sequence variants as recommended by the Human Genome Variation Society.

\section{RNA extraction and real-time qPCR}

Total RNA was extracted from whole blood using TRIzol reagent (Invitrogen, Carlsbad, CA, USA) according to the manufacturer's instructions. Next, $0.5 \mu \mathrm{g}$ of total RNA was reverse transcribed into cDNA using a high capacity cDNA reverse transcription kit (Applied Biosystems). Real-time qPCR was performed using a QuantiTect SYBR Green PCR kit (Qiagen) in an ABI PRISM 7900 HT system (Applied Biosystems). The sequences of the primers were as follows: PTEN-F, CAGAAAGACTTGAAGGCGTAT; PTEN-R, AACGGCTGAGGGAACTC; KILLIN-F: AAAAGAATTCCGGGGCTGGCGCTTGGGG; KILLIN-R: AAAAGCGGCCGCGTCCTT TGGCTTGCTCTTAGG; GAPDH-F, GAAGGTGAAGGTCGGAGT; GAPDH-R, GAAGAT GGTGATGGGATTTC. Expression levels of PTEN and KILLIN mRNA were normalized to glyceraldehyde-3-phosphate dehydrogenase (GAPDH). Fold change in PTEN/KILLIN expression was calculated by the equation $2^{-\Delta \Delta \mathrm{Ct}}$. $\Delta \mathrm{Ct}$ was calculated by subtracting the $\mathrm{Ct}$ values of $G A P D H$ from the $\mathrm{Ct}$ values of the genes. $\Delta \Delta \mathrm{Ct}$ was then calculated by subtracting $\Delta \mathrm{Ct}$ of the control from $\Delta \mathrm{Ct}$ of breast cancer. Real-time qPCR was performed in triplicate. 


\section{Methylation-sensitive restriction enzyme digestion and MSRED-qPCR}

Methylation-sensitive restriction enzyme digestion followed by qPCR (MSRED-qPCR) assays were performed, as described previously. ${ }^{13}$ In brief, 100 ng of genomic DNA from either ethylenediaminetetraacetic acid blood or plasma samples was digested in a $40 \mu \mathrm{L}$ reaction volume with $30 \mathrm{U}$ of the methylation-sensitive restriction enzyme, BstU1 (New England BioLabs, Hitchin, UK) at $60^{\circ} \mathrm{C}$ for 16 hours. To ensure complete enzyme digestion, a positive and a negative control digestion containing $30 \mathrm{ng}$ of completely methylated or unmethylated control DNA (EpiTect Control DNA; Qiagen) were run in parallel. After digestion, the same amount of digested or undigested DNA along with control digestion was subjected to qPCR using a QuantiTect SYBR Green PCR kit in an ABI 7900 HT system. The primer sequence for the KILLIN/ PTEN promoter was F- GTTGTAGTTTTAGGGAGGGGGT; R-CTACTTCTCCTCAACAACCAAAAAC. Each reaction was performed in a final volume of $20 \mu \mathrm{L}$ containing digested (1.3 $\mu \mathrm{L})$ or undigested $(1 \mu \mathrm{L}) \mathrm{DNA}, 500 \mathrm{nM}$ of each primer, and $1 \times$ SYBR Green PCR Master Mix (Qiagen). At the end of the PCR cycles, melting curve analyses were performed to validate the specific PCR product. Relative expression of methylated DNA was expressed as $2^{\Delta \mathrm{Ct} \text { (undigest-digest) }} \Delta \mathrm{Ct}_{\text {(undigest-digest) }}$ was calculated by subtracting the $\mathrm{Ct}$ values of plasma DNA from the $\mathrm{Ct}$ values of undigested DNA. Given that the Ct of undigest should be less than or equal to the $\mathrm{Ct}$ of digest, the expression level ranged from 1 to 0 . Each sample was run in duplicate for analysis. For $100 \%$ digestion efficiency, the relative expression level of completely unmethylated control (CTRL) DNA (2 $\left.{ }^{\triangle \mathrm{Ct}(\mathrm{CTRL} \text { undigest-CTRLdigest) }}\right)$ must be close to zero, whereas the level of completely methylated control must be 1 .

\section{Statistical analysis}

The significance of PTEN and KILLIN expression levels in blood was determined using the Mann-Whitney $U$ test. The statistical significance of plasma methylated KILLIN DNA levels was also determined by the Mann-Whitney $U$ test. The correlation between PTEN and KILLIN gene expression was determined by Spearman's rank correlation coefficient. All $P$-values were two-sided and a value $<0.05$ by GraphPad Prism 5 software (GraphPad Software, La Jolla, CA, USA) was considered to be statistically significant.

\section{Results}

\section{Patient characteristics}

A total of 23 patients with breast and/or thyroid cancer were recruited. The mean age at diagnosis of breast cancer was
51.4 (range 33-74) years and that of thyroid cancer was 43.84 (range 19-74) years. The mean age of the healthy controls was 49.7 years. The patient characteristics were shown in Table 1.

\section{PTEN mutation screening by full gene sequencing}

Based on our PTEN sequencing results, no PTEN coding mutations were found. Only four single nucleotide polymorphisms were identified, including c. $1-9 \mathrm{C}>\mathrm{G}$, c. $80-96 \mathrm{~A}>\mathrm{G}$, c. $1026+32 \mathrm{~T}>\mathrm{G}$, and c. $1212+75 \mathrm{~T}>\mathrm{A}$, which were reported in the Single Nucleotide Polymorphism Database of the National Center of Biotechnology Information (Table 2).

\section{Downregulated expression of KILLIN but not PTEN}

We examined the expression levels of PTEN and KILLIN using qPCR in blood samples from 23 patients and 20 healthy controls. Our results show that PTEN gene expression was higher in cancer patients when compared with healthy controls (Figure 1A). On the other hand, expression of KILLIN was significantly decreased in patients when compared with healthy controls (Figure 1B). However, there is no direct correlation between PTEN and KILLIN mRNA expression (Figure 1C). Interestingly, when we stratified patients into those with breast cancer only, thyroid cancer only, and both breast cancer and thyroid cancer, the expression level of PTEN was significantly increased in those with breast cancer and thyroid cancer, and in those with breast cancer only, when compared with healthy controls (Figure 2A). Also, there was a decreasing trend of KILLIN expression levels in patients with breast cancer and thyroid cancer, those with breast cancer only, and those with thyroid cancer only relative to healthy controls (Figure 2B).

\section{Quantitative analysis of methylated KILLIN DNA in the plasma of patients}

To investigate whether downregulation of expression is associated with hypermethylation of the KILLIN/PTEN promoter, we developed a simple methylation-sensitive restriction enzyme digestion and real-time quantitative assay to quantify the methylated KILLIN DNA in the patients' blood samples. Initially, methylated KILLIN DNA levels in blood samples from the 23 patients and 20 healthy controls were assessed. Our results indicated that there was no significant difference in blood levels of methylated DNA between cancer patients and healthy controls ( $P=0.111$; Figure $3 \mathrm{~A})$. We also assessed the level of methylated KILLIN/PTENDNA in the plasma samples. 
Table I Patient characteristics

\begin{tabular}{|c|c|c|c|c|c|c|}
\hline Case number & $\begin{array}{l}\text { Age at diagnosis } \\
\text { of } B C \text {, years }\end{array}$ & BC type & $\begin{array}{l}\text { Family history } \\
\text { of BC }\end{array}$ & $\begin{array}{l}\text { Age at diagnosis } \\
\text { of TC, years }\end{array}$ & TC type & $\begin{array}{l}\text { Family history } \\
\text { of TC }\end{array}$ \\
\hline \multicolumn{7}{|l|}{$\mathrm{BC}$ and $\mathrm{TC}$} \\
\hline PMHI30I & 33 & IDC & No & 21 & Colloid nodule & NA \\
\hline TWH5I70I & 40 & IDC + ILC & NA & 26 & FTC & NA \\
\hline TWH5I90I & 47 & IDC & No & 35 & FTC & NA \\
\hline TWH4970I & 42 & IDC & No & 43 & NA & No \\
\hline PTEN20I & 35 & $\mathrm{DC}$ & No & 44 & PTC & No \\
\hline PTEN90I & 64 & IDC & NA & 46 & FTC & NA \\
\hline PTENIIOI & 74 & IDC & NA & 57 & FTC & NA \\
\hline HKSHIIOI & 51 & IDC + ILC & NA & 60 & PTC & NA \\
\hline PTENIO0I & 66 & NA & No & 70 & PTC & No \\
\hline PTENIOI & 70 & IDC & Yes & 70 & PTC & NA \\
\hline UCH70I & 73 & IDC & No & 74 & Medullary & NA \\
\hline $\mathrm{UCH} 20 \mathrm{I}$ & 38 & IDC & No & 38 & PTC & NA \\
\hline QEH60I & 52 & IDC & NA & 52 & PTC & No \\
\hline QMH780I & 71 & DCIS & No & 20 & NA & No \\
\hline TWH4230I & 38 & IDCII (L); DCIS (R) & No & 38 & PTC & No \\
\hline TWH5620I & 50 & IDCII & NA & 42 & PTC & NA \\
\hline \multicolumn{7}{|l|}{ TC only } \\
\hline TWH46303 & Not applicable & Not applicable & NA & 19 & PTC & Yes \\
\hline TWH46302 & Not applicable & Not applicable & NA & 44 & PTC & Yes \\
\hline TWH3640I & Not applicable & Not applicable & Yes & 34 & PTC & NA \\
\hline \multicolumn{7}{|l|}{ BC only } \\
\hline TWH3690I & 40 & IDC & No & Not applicable & Not applicable & Yes \\
\hline TWH4IOI & 44 & IDC & Yes & Not applicable & Not applicable & Yes \\
\hline TWH4630I & 50 & IDC (L); DCIS (R) & No & Not applicable & Not applicable & Yes \\
\hline TWH5090I & 50 & IDC & NA & Not applicable & Not applicable & Yes \\
\hline
\end{tabular}

Abbreviations: BC, breast cancer; TC, thyroid cancer; NA, not available; IDC, invasive ductal carcinoma; DCIS, ductal carcinoma in situ; ILC, invasive lobular carcinoma; DC, ductal carcinoma; PTC, papillary thyroid carcinoma; FTC, follicular thyroid carcinoma; (L), left breast; (R), right breast; PMH, Princess Margaret Hospital; TWH, Tung Wah Hospital; HKSH, Hong Kong Sanatorium and Hospital; UCH, United Christian Hospital; QEH, Queen Elizabeth Hospital; QMH, Queen Mary Hospital.

Our results demonstrated that plasma levels of methylated KILLIN DNA in the 23 cancer patients were significantly increased when compared with those in controls $(P<0.05$; Figure 3B), suggesting hypermethylation of the KILLIN/PTEN promoter in these patients, and significant negative correlation between blood level of KILLIN mRNA expression and plasma level of methylated KILLIN DNA of the 43 subjects including 20 healthy controls and 23 cancer patients (Spearman $r=-0.58$, $P<0.0001$; Figure 4).

\section{Discussion}

In this study, we found that KILLIN gene expression but not that of PTEN, was significantly decreased in blood samples

Table 2 PTEN polymorphisms in patients with cancer of the breast and/or thyroid

\begin{tabular}{lll}
\hline Location & Variant & NCBI ref SNP \\
\hline $5^{\prime} U T R$ & c. I-9C $>$ G & rs II 202592 \\
Intron I & c. $80-96 \mathrm{~A}>\mathrm{G}$ & rs 1903858 \\
Intron 8 & c. $1026+32 \mathrm{~T}>\mathrm{G}$ & $\mathrm{rs} 555895$ \\
Intron 9 & c. $1212+75 T>\mathrm{A}$ & $\mathrm{rs} 74535369$
\end{tabular}

Abbreviations: $\mathrm{NCBI}$, National Center of Biotechnology Information; ref, reference; SNP, single nucleotide polymorphism; UTR, untranslated region. from Chinese women with breast and/or thyroid cancers. We then quantified plasma methylated KILLIN/PTEN DNA levels in these patients and demonstrated that they were significantly elevated. To the best of our knowledge, this is the first report to show increased plasma levels of methylated KILLIN DNA in such patients, suggesting hypermethylation of the KILLIN/PTEN promoter in breast and thyroid cancer.

Given that variants c. $80-96 \mathrm{~A}>\mathrm{G}, \mathrm{c} .1026+32 \mathrm{~T}>\mathrm{G}$, and $1212+75 \mathrm{~T}>\mathrm{A}$ were identified in most of our subjects and the fact that they were located far away from the PTEN exons, these variants might be presumed to have no effect on normal PTEN function. ${ }^{2}$ It is unclear whether variant c. $1-9 \mathrm{C}>\mathrm{G}$ would have any effect on PTEN expression in breast and thyroid carcinoma. This variant is shown in the Single Nucleotide Polymorphism Database from the National Centre of Biotechnology Information with a noted frequency of 0.024 , and has not been reported in carcinoma. However, overexpression of PTEN has been shown to be associated with this variant in type 2 diabetes in the Japanese population. ${ }^{7}$ The nucleotide sequence around the AUG initiation codon can influence recognition of the ribosome and affect the efficiency of translation. It was suggested that 

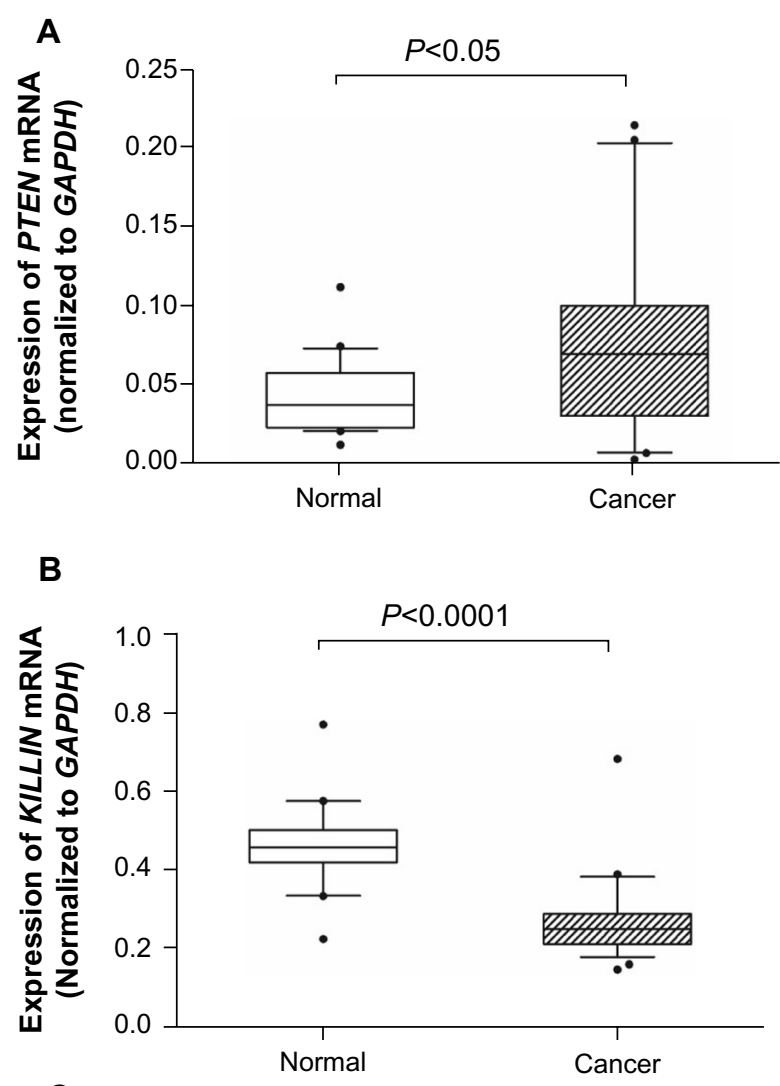

C

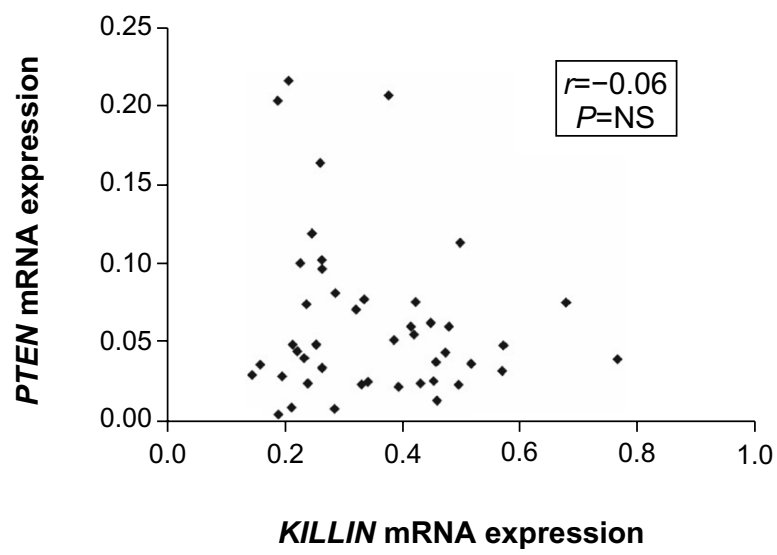

Figure I PTEN and KILLIN expression in cancer patients.

Notes: Gene expression of (A) PTEN and (B) KILLIN in blood samples from healthy normal subjects $(n=20)$ and patients with cancer of the breast and/or thyroid $(n=23)$. Expression of mRNA was normalized to GAPDH. The lines inside the boxes denote the medians. The boxes mark the interval between the 25 th and 75 th percentiles. The whiskers denote the interval between the 10th and 90th percentiles. Statistical significance of differences was analyzed using Mann-Whitney $U$ tests. (C) Correlation between PTEN and KILLIN mRNA expression (Spearman rank correlation, $r=-0.06$, $P=N S$, not significant).

Abbreviations: NCBI, National Center of Biotechnology Information; SNP, single nucleotide polymorphism.

substitution of the $\mathrm{G}$ residue at position 9 results in greater homology of the PTEN gene sequence, ctcccagacATGa, to the Kozak sequence $\operatorname{gccgcc}(\mathrm{a} / \mathrm{g}) \mathrm{ccATGg}$, which has been reported to enhance translation in mammalian cells. ${ }^{7,14}$ The prevalence of c. $1-9 \mathrm{C}>\mathrm{G}$ in diabetic patients was reported to

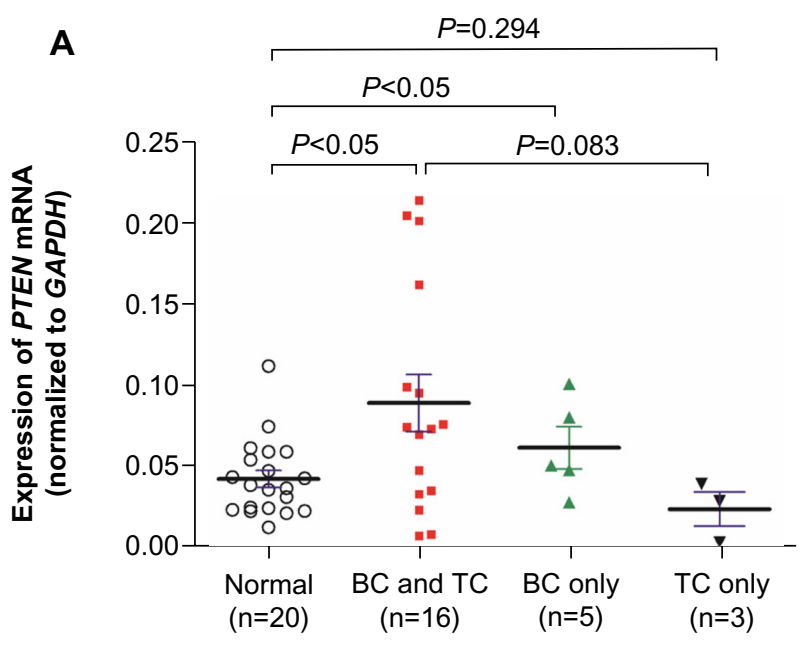

B

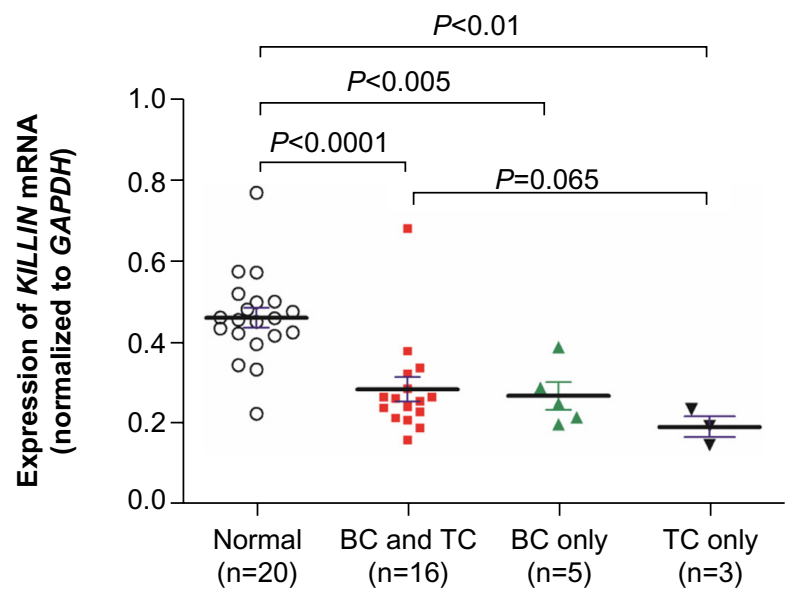

Figure 2 Expression levels of PTEN and KILLIN in patients with breast and thyroid cancer.

Notes: Gene expression of (A) PTEN and (B) KILLIN in blood samples from healthy normal subjects $(n=20)$, and patients with breast and thyroid cancer $(n=16)$, breast cancer only $(n=4)$, and thyroid cancer only $(n=3)$.

Abbreviations: BC, breast cancer; TC, thyroid cancer.

be $14 \%(15 / 107)$ for both the heterozygous and homozygous variants, whereas only $5 \%(5 / 100)$ of control subjects carried the heterozygous variant but not the homozygous variant. ${ }^{7}$ In our study, three of $18(16.6 \%)$ patients carried the heterozygous variant and no homozygous variant was found. Due to the small sample size and lack of comparison with control subjects, no association between the polymorphism and our cancer patients could be identified. The pathogenicity of this variant in breast and thyroid cancers remains to be elucidated and confirmed by protein expression and functional assays. However, the broad phenotypic spectrum of multiple hamartoma syndromes makes diagnosis of CS complicated, and hence recruitment of suitable subjects for research is difficult. ${ }^{1,2,6,15-17}$ CS-like patients, with features of CS but not meeting the strict diagnostic criteria, might not have PTEN mutation. ${ }^{2,6,16}$ Although breast and thyroid 


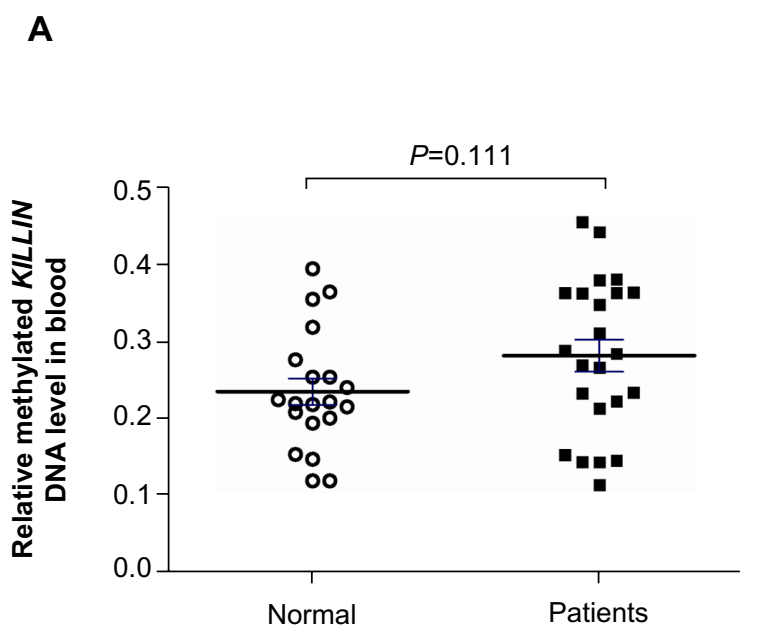

B

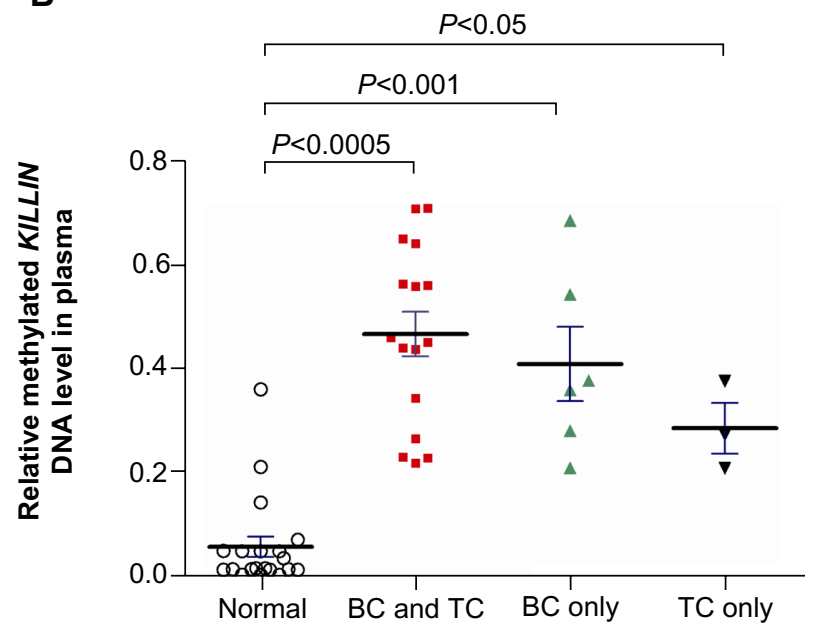

Figure 3 Methylated KILLIN DNA expression in plasma samples from healthy normal subjects and patients with breast and/or thyroid cancer.

Notes: Quantitative analysis of methylated KILLIN/PTEN DNA in (A) blood samples from healthy normal subjects $(n=20)$ and patients $(n=23)$ and in $(B)$ plasma samples by methylation-sensitive restriction enzyme digestion followed by qPCR. Scatter plots for plasma levels of methylated KILLIN DNA in healthy normal subjects $(n=20)$ and patients with breast and thyroid cancer $(n=16)$, breast cancer $(n=4)$, and thyroid cancer $(n=3)$. Horizontal lines denote the medians. Statistically significant differences were determined using the Mann-Whitney $U$ test, $P<0.0001$.

Abbreviations: $\mathrm{BC}$, breast cancer; $\mathrm{qPCR}$, quantitative polymerase chain reaction; TC, thyroid cancer.

cancers are the most common manifestation of CS, renal cell and endometrial carcinoma could be included in the patient selection criteria to increase the chances of finding PTEN mutations. ${ }^{6,8,16-18}$ In addition to the coding region, it is suggested that the promoter region is an important site of PTEN analysis. A screen of 119 CS patients negative for PTEN mutation at the coding region showed that $10 \%$ had mutations located at the promoter region between -1344 and $-745 \mathrm{bp}$ upstream of the translation start codon. ${ }^{4}$ It was estimated that mutations at the promoter might result in post-translational modifications or targeted PTEN degradation, thereby leading to impaired protein expression. ${ }^{4}$ Other than PTEN,

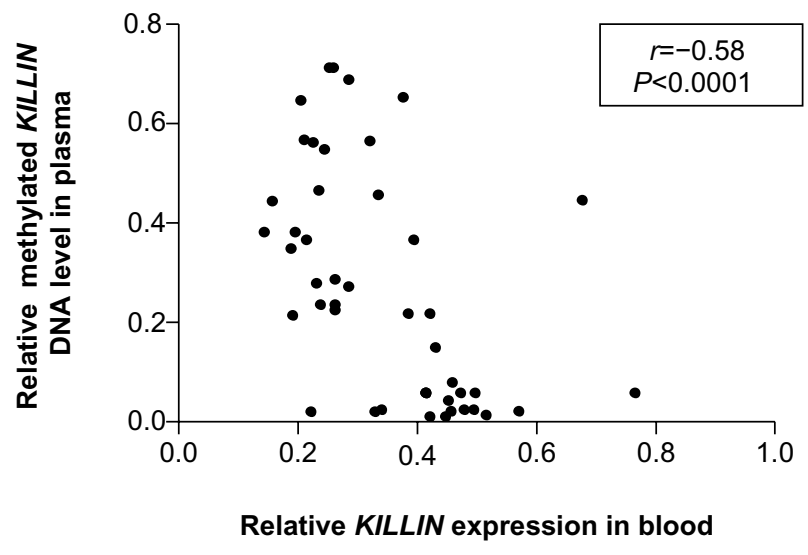

Figure 4 Correlation analysis between blood level of KILLIN mRNA expression and plasma level of methylated KILLIN DNA in 20 healthy normal subjects and 23 cancer patients (Spearman rank correlation, $r=-0.58, P<0.000$ I).

succinate dehydrogenase genes might be alternative markers for CS/CS-like syndromes. ${ }^{6,17}$ Succinate dehydrogenase is a mitochondrial enzyme complex that participates in the electron transport chain and Kreb's cycle. Like PTEN, succinate dehydrogenase also has a tumor suppressor function, and negatively regulates the Akt and mitogen-activated protein kinase signaling pathway. ${ }^{19}$ One study showed that $13.5 \%$ of PTEN mutation-negative CS/CS-like patients had germline succinate dehydrogenase complex subunit B (SDHB) and succinate dehydrogenase complex subunit D (SDHD) mutations. ${ }^{6}$ Patients with succinate dehydrogenase mutations had increased levels of phosphorylated Akt and mitogen-activated protein kinase, causing dysregulation of apoptosis. Higher frequencies of breast, thyroid, and renal cell carcinomas were observed in succinate dehydrogenase mutation carriers than PTEN mutation carriers. ${ }^{6}$

Apart from PTEN, KILLIN is another important gene that might be implicated in breast cancer. One recent study offers an intriguing explanation for some of the families with PTEN wild-type CS and Cowden-like syndrome. ${ }^{10}$ The authors of that study examined peripheral lymphocytes from patients with CS or Cowden-like syndrome for hypermethylation of the PTEN promoter. ${ }^{10}$ Unexpectedly, they discovered that although a significant proportion of patients had hypermethylation of the PTEN promoter, silencing of PTEN was not found. Bennett et al investigated a relatively new gene known as KILLIN. KILLIN has recently been identified and little is known about its function or its role in cancer. They found that KILLIN is indeed transcribed in the opposite, ie, antisense, strand relative to PTEN and shares the same promoter as PTEN. Thus, they postulated that the methylation changes in their patient samples were indeed regulating KILLIN expression and not that of PTEN. They demonstrated 
that patients with KILLIN/PTEN promoter hypermethylation have significantly reduced KILLIN gene expression levels compared with controls. ${ }^{10}$ Since there is only an association between downregulated KILLIN expression and hypermethylation of the KILLIN/PTEN promoter, PTEN expression was not affected by promoter hypermethylation. We speculate that other regulatory mechanisms may be involved in PTEN expression, specifically in the development of breast cancer.

We believe that our finding of increased plasma methylated KILLIN/PTENDNA in patients with thyroid and breast cancers relative to those with either of these cancers alone might have important clinical implications. One possible clinical scenario would be if a female patient has been diagnosed with a follicular-derived thyroid carcinoma and is also found to have raised plasma methylated KILLIN/ PTEN DNA. Our results suggest that such a patient has a relatively higher chance of developing breast cancer in the future and so would benefit from breast cancer screening. In other words, methylated KILLIN/PTEN DNA in plasma could be used as a diagnostic marker for patients with an increased lifetime risk of developing both cancers. However, a much larger longitudinal study would be needed to confirm this.

\section{Conclusion}

Taken together, no correlation between PTEN mutations and cancer of the breast and/or thyroid was found in this study. Nonetheless, hypermethylation of the KILLIN/PTEN promoter could have contributed to the development of these cancers in those patients without identifiable PTEN mutations. In this regard, we showed that plasma methylated KILLIN/PTEN DNA was significantly increased, suggesting hypermethylation of the KILLIN/PTEN promoter in patients with breast and/or thyroid cancers. Because our sample size was small, further validation in a larger sample size is required to confirm the potential diagnostic usefulness of this methylated DNA marker.

\section{Acknowledgment}

We sincerely thank the Dr Ellen Li Charitable Foundation for its support.

\section{Disclosure}

The authors report no conflict of interests in this work.

\section{References}

1. Liaw D, Marsh DJ, Li J, et al. Germline mutations of the PTEN gene in Cowden disease, an inherited breast and thyroid cancer syndrome. Nat Genet. 1997;16:64-67.

2. Eng C. PTEN: one gene, many syndromes. Hum Mutat. 2003;22: 183-198.

3. Carroll BT, Couch FJ, Rebbeck TR, Weber BL. Polymorphisms in PTEN in breast cancer families. J Med Genet. 1999;36:94-96.

4. Zhou XP, Waite KA, Pilarski R, et al. Germline PTEN promoter mutations and deletions in Cowden/Bannayan-Riley-Ruvalcaba syndrome result in aberrant PTEN protein and dysregulation of the phosphoinositol3-kinase/Akt pathway. Am J Hum Genet. 2003;73:404-411.

5. Tamura M, Gu J, Matsumoto K, Aota S, Parsons R, Yamada KM. Inhibition of cell migration, spreading, and focal adhesions by tumor suppressor PTEN. Science. 1998;280:1614-1617.

6. Ni Y, Zbuk KM, Sadler T, et al. Germline mutations and variants in the succinate dehydrogenase genes in Cowden and Cowden-like syndromes. Am J Hum Genet. 2008;83:261-268.

7. Ishihara H, Sasaoka T, Kagawa S, et al. Association of the polymorphisms in the $5^{\prime}$-untranslated region of PTEN gene with type 2 diabetes in a Japanese population. FEBS Lett. 2003;554:450-454.

8. Hu YC, Lam KY, Tang JC, Srivastava G. Mutational analysis of the PTEN/MMAC1 gene in primary oesophageal squamous cell carcinomas. Mol Pathol. 1999;52:353-356.

9. Cho YJ, Liang P. Killin is a p53-regulated nuclear inhibitor of DNA synthesis. Proc Natl Acad Sci U SA. 2008;105:5396-5401.

10. Bennett KL, Mester J, Eng C. Germline epigenetic regulation of KILLIN in Cowden and Cowden-like syndrome. JAMA. 2010;304: 2724-2731.

11. Kwong A, Wong LP, Wong HN, et al. A BRCA2 founder mutation and seven novel deleterious BRCA mutations in southern Chinese women with breast and ovarian cancer. Breast Cancer Res Treat. 2009;117: 683-686.

12. Kwong A, Wong LP, Wong HN, et al. Clinical and pathological characteristics of Chinese patients with BRCA related breast cancer. HUGOJ. 2009;3:63-76

13. Ng EK, Leung CP, Shin VY, et al. Quantitative analysis and diagnostic significance of methylated SLC19A3 DNA in the plasma of breast and gastric cancer patients. PLoS One. 2011;6:e22233.

14. Kozak M. At least six nucleotides preceding the AUG initiator codon enhance translation in mammalian cells. J Mol Biol. 1987;196: 947-950.

15. Bonneau D, Longy M. Mutations of the human PTEN gene. Hum Mutat. 2000;16:109-122.

16. Pezzolesi MG, Platzer P, Waite KA, Eng C. Differential expression of PTEN-targeting microRNAs miR-19a and miR-21 in Cowden syndrome. Am J Hum Genet. 2008;82:1141-1149.

17. Zbuk KM, Patocs A, Shealy A, Sylvester H, Miesfeldt S, Eng C. Germline mutations in PTEN and SDHC in a woman with epithelial thyroid cancer and carotid paraganglioma. Nat Clin Pract Oncol. 2007;4:608-612.

18. Mutter GL, Lin MC, Fitzgerald JT, et al. Altered PTEN expression as a diagnostic marker for the earliest endometrial precancers. J Natl Cancer Inst. 2000;92:924-930.

19. Eng C, Kiuru M, Fernandez MJ, Aaltonen LA. A role for mitochondrial enzymes in inherited neoplasia and beyond. Nat Rev Cancer. 2003;3: 193-202. 


\section{Supplementary material}

Table SI Sequence of polymerase chain reaction and sequencing primers for PTEN gene

\begin{tabular}{|c|c|c|}
\hline Exon & Forward primer sequence $\left(5^{\prime}\right.$ to $\left.3^{\prime}\right)$ & Reverse primer sequence $\left(5^{\prime}\right.$ to $\left.3^{\prime}\right)$ \\
\hline I & ATTTCCATCCTGCAGAAGAAGC & GCAACCAGGCAAGAGTTCCGT \\
\hline 2 & TTTATTACTCCAGCTATAGTGGG & CCATTAGGTACGGTAAGCCAAA \\
\hline 3 & CCATAGAAGGGGTATTTGTTGG & GGACTTCTTGACTTAATCGGTTT \\
\hline 4 & TAAACACAGCATAATATGTGTCAC & ATGTATCTCACTCGATAATCTGG \\
\hline 5 & TTAAGTTTGTATGCAACATTTCTA & GTATATACACATACATCAAAACATC \\
\hline 6 & GTATATATGTTCTTAAATGGCTA & CTTCAGAAATATAGTCTCCTGCAT \\
\hline 7 & GATACAGAATCCATATTTCGTGTA & GTAAGCAAAACACCTGCAGATC \\
\hline 8 & CAAATGTTTAACATAGGTGACAGA & CTGCTACGTAAACACTGCTTCGA \\
\hline $8 \mathrm{~S}$ & GACAAAATGTTTCACTTTTGGGT & \\
\hline 9 & TAAAGATCATGTTTGTTACAGTGC & TCTGACACAATGTCCTATTGCC \\
\hline 9S & & TTCATGGTGTTTTATCCCTCTTG \\
\hline
\end{tabular}

Abbreviation: $\mathrm{S}$, sequencing primer.

\section{Publish your work in this journal}

OncoTargets and Therapy is an international, peer-reviewed, open access journal focusing on the pathological basis of all cancers, potential targets for therapy and treatment protocols employed to improve the management of cancer patients. The journal also focuses on the impact of management programs and new therapeutic agents and protocols on patient perspectives such as quality of life, adherence and satisfaction. The manuscript management system is completely online and includes a very quick and fair peer-review system, which is all easy to use. Visit http://www.dovepress.com/testimonials.php to read real quotes from published authors. 